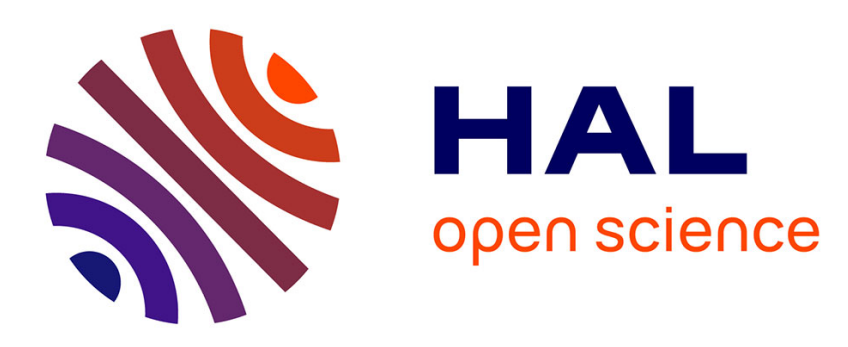

\title{
Low Complexity Versatile Video Coding (VVC) for Low Bitrate Applications
}

\author{
Mourad Aklouf, Marc Leny, Frédéric Dufaux, Michel Kieffer
}

\section{To cite this version:}

Mourad Aklouf, Marc Leny, Frédéric Dufaux, Michel Kieffer. Low Complexity Versatile Video Coding (VVC) for Low Bitrate Applications. 8th European Workshop on Visual Information Processing (EUVIP 2019), Oct 2019, Rome, Italy. pp.22-27, 10.1109/EUVIP47703.2019.8946261 . hal-02299789

\section{HAL Id: hal-02299789 \\ https://hal.science/hal-02299789}

Submitted on 10 Jan 2020

HAL is a multi-disciplinary open access archive for the deposit and dissemination of scientific research documents, whether they are published or not. The documents may come from teaching and research institutions in France or abroad, or from public or private research centers.
L'archive ouverte pluridisciplinaire HAL, est destinée au dépôt et à la diffusion de documents scientifiques de niveau recherche, publiés ou non, émanant des établissements d'enseignement et de recherche français ou étrangers, des laboratoires publics ou privés. 


\section{LOW COMPLEXITY VERSATILE VIDEO CODING (VVC) FOR LOW BITRATE APPLICATIONS}

\author{
Mourad AKLOUF, Marc LENY
}

\author{
\{maklouf, mleny\}@ektacom.com, \\ EKTACOM, \\ 91940 Les Ulis, France.
}

\begin{abstract}
The new Versatile Video Coding (VVC) standard is currently under development, targeting more efficient compression, especially for $4 \mathrm{~K}$ and $8 \mathrm{~K}$ contents. Nevertheless, some application scenarios still require low resolution and low bitrate encoding, e.g., live video streaming over unstable bandwidth-limited wireless networks. In addition, embedded applications also require low complexity and low power consumption solutions. As VVC is not designed for these conditions, it may not necessarily achieve the optimal tradeoff between complexity and compression efficiency. This paper proposes an optimization framework to determine the efficiency at lowresolution and low-bitrate of some of the new coding tools introduced in VVC. We show that in such coding conditions, significant reduction in terms of encoding complexity (up to $56 \%$ at $384 \times 216$ resolution) may be obtained by disabling some of the VVC coding tools, with a negligible impact in terms of compression efficiency (less than $1.88 \%$ increase in $\mathrm{BD}_{\text {rate }}$ ).
\end{abstract}

Index Terms - Versatile Video Coding, video compression, optimization, low complexity, low resolution, low bitrate

\section{INTRODUCTION}

Video traffic over Internet is continuously increasing. Video is foreseen to represent $82 \%$ of all consumer Internet traffic by $2021,26 \%$ of which will be Internet video-to-TV traffic. Moreover, Content Delivery Network (CDN) traffic will carry $71 \%$ of all Internet traffic by $2021[1]$.

This congestion is mainly due to users expecting better quality of experience, including High Definition (HD) and Ultra-High Definition (UHD) video content. UHD, including $3840 \times 2160(4 \mathrm{~K})$ and $7680 \times 4320(8 \mathrm{~K})$ formats, is expected to become mainstream. For instance, $4 \mathrm{~K}$ TVs are already available since 2014 , and $8 \mathrm{~K}$ consumer TVs are expected in 2020. Even portable devices are expected to handle UHD in the next few years, due to increasing hardware power and efficiency of IP networks. In this context, the need for new efficient video compression tools is clear, on one hand to decrease the load on transmission channels and storage servers, and on the other hand to provide high-quality content to end-users.

The High-Efficiency Video Coding (HEVC) standard [2] is the latest standard developed by the Joint Collaborative Team on Video Coding (JCT-VC) of VCEG and MPEG. It is effectively deployed worldwide and compares favorably with competing solutions [3]. Nevertheless, more efficient video compression techniques are still desired. With this goal in mind, the new Versatile Video Coding (VVC) standard is currently under development by the Joint Video

\author{
Frederic DUFAUX, Michel KIEFFER
}

\{frederic.dufaux, michel.kieffer\}@12s.centralesupelec.fr, L2S - CentraleSupelec - CNRS - Univ Paris Sud, 91192 Gif-sur-Yvette, France.

Exploration Team (JVET) of VCEG and MPEG and is expected to be finalized by 2020 [4].

While the above standards are mainly focused on HD and UHD content, efficient video compression solutions are also requested for streaming Standard Definition (SD) video (or even lower resolutions) over unstable bandwidth-limited networks. Indeed, whereas HD video is now the standard on the Internet, low-resolution contents are still used, especially 480p and 360p. In this paper, we specifically consider use cases such as the acquisition and live streaming of low-resolution (less than HD) sport events (e.g., car races and sailing races) over relatively unstable wireless networks including LTE and satellite transmissions. For such use cases, transmission bandwidth when using common wireless networks is typically in the range of 50 to $700 \mathrm{kbps}$. Moreover, low computational complexity and low power consumption solutions are highly desired for embedded systems.

As current VVC developments are not designed for such use cases, some of the design choices for HD and UHD may not be optimal for lower resolutions and low-bitrates. More precisely, a variety of encoding tools available at the VVC encoder may entail a significant burden in terms of computational complexity. Moreover, some of these tools may not be suited in the case of low resolution and low bitrate scenarios. In particular, the cost of syntax elements to signal the activation of these tools may represent a non-negligible overhead at low bitrates.

The contribution of this paper is two fold. We first propose an optimization framework with the objective to tune VVC for lowresolution and low-bitrate scenarios. More specifically, we investigate the usefulness of some of the new coding tools in VVC. Second, we experimentally show that significant complexity reduction can be achieved (up to $56.06 \%$ reduction for Johnny sequence at $384 \times 216$ resolution) by disabling some of these tools, while preserving coding efficiency (less than $1.88 \%$ increase in $\mathrm{BD}_{\text {rate }}$ ). To the best of our knowledge, this is the first study to investigate VVC at low resolutions and low-bitrates, and to report such complexity reduction results.

The remaining of this paper is structured as follow. Some related works are first reviewed in Section 2. Section 3 provides a brief overview of the technical features of the VVC standard and the coding tools in the VVC Test Model (VTM). The problem formulation and proposed optimization framework are introduced in Section 4. Experimental results are reported in Section 5. Finally, we draw some conclusions in Section 6. 


\section{RELATED WORKS}

The problem of encoder optimization has already been largely addressed in the literature. In particular, many proposals have considered the simplification of the Rate Distortion Optimization (RDO) process of the HEVC encoder. Targeting the Coding Unit (CU) size and depth decision, an algorithm to quickly determine the CU size has been proposed in [5] for both Intra and Inter modes. To reduce the number of Intra mode candidates, [6] uses the coding information of the neighboring blocks. Conversely, [7] introduces a decision algorithm, which compute the dominant edge of the Prediction Unit (PU) using the local samples. Targeting the Motion Vectors (MVs) search in Inter prediction, [8] presents an heuristic to determine the $\mathrm{PU}$ partition by checking all CUs among the neighboring NxN partition.

All the previous approaches make algorithmic modifications to the non-normative encoding process. In this paper, our aim is to identify coding tools which can be ignored in low-bitrate use cases, with a greatly reduced complexity and a preserved coding efficiency. This could lead to the definition of application-oriented profiles, where some tools are automatically disabled in the highlevel syntax.

\section{VERSATILE VIDEO CODING (VVC)}

VVC [9] is a new video coding standard designed by JVET, which goal is to provide significant improvements in compression performance over the existing HEVC standard, with a target of $50 \%$ bitrate saving. VVC is expected to deliver UHD services ( $4 \mathrm{~K}$ and $8 \mathrm{~K}$ ) at approximately the same bit rates used today to carry HDTV. The requirements for VVC include capabilities of encoding $4 \mathrm{~K}$ and $8 \mathrm{~K}$ sequences of up to $120 \mathrm{fps}$ [10]. In what follows, some of the coding tools in VTM5.0 are overviewed [11].

\subsection{Partitioning}

Each frame is divided into a sequence of coding tree units (CTUs) just as in the HEVC standard, although the maximum size of the Luma CTU is up to $128 \times 128$. For each CTU, a Quad-Tree with nested Multi-Type Tree (MTT) using Binary and Ternary splitting structures is used (QTBT-TT). The CTU is first partitioned recursively using a quad-tree structure into square shapes. Then, the quad-tree leaf nodes can be further partitioned horizontally or vertically by a binary or ternary splitting structure. The final nodes are called Coding Units (CUs). They have either a square or rectangular shape and are used directly for prediction and residual coding. Lastly, I-slices can have separate block tree structures for Luma and Chroma (DualTree).

\subsection{Intra-Picture Prediction}

VTM5.0 supports 65 angular intra-prediction modes, in addition to the planar and DC modes. Some conventional angular modes are replaced with wide-angle intra-prediction modes for the non-square blocks. A Multiple Reference Line (MRL) intra prediction is also proposed to use two additional lines (reference line 1 and reference line 3) in angular prediction. VTM5.0 also extends the Most Probable Modes (MPM) list to 6 candidates.

VTM5.0 introduces three new ways of Intra predicting a block: (1) the Cross-Component Linear Model (CCLM) prediction mode, in which the Chroma samples are predicted based on the reconstructed Luma samples of the same CU, using a linear model; (2) The
Intra Sub-Partitions (ISP) where the Luma CB is vertically or horizontally divided into 2 or 4 sub-partitions. All sub-partitions share the same intra mode, however the processing is performed gradually sub-partition by sub-partition downwards (horizontal split) or rightwards (vertical split), so each one uses the previous reconstructed samples to generate the prediction of the current sub-partition; (3) The Matrix-based Intra Prediction (MIP) takes one line of reconstructed neighboring samples, from the left and above blocks as input vectors, and after some pre-processing, performs linear interpolation in the vertical and horizontal directions.

\subsection{Inter-Picture Prediction}

Motion prediction is performed at a sub-CU level to improve the precision. VTM5.0 supports currently a Sub-Pu Temporal Motion Vector Prediction (SbTMVP). Furthermore, an AFFine motion compensation prediction (AFF) can be applied to cope with irregular motions like zoom in/out and rotation, where a sub-block is described by two or three motion vectors.

The bi-prediction mode is extended beyond simple weighted averaging, by using up to five predefined weights (Generalized Bi-prediction (GBI)) and a pixel level motion refinement (BiDirectional Optical Flow (BDOF)) may be performed on top of it. In order to increase the accuracy of the MVs of the merge mode, a refined operation may be performed around the initial MVs in both reference picture lists L0 and L1 using the Decoder side Motion Vector Refinement (DMVR). Motion vectors are stored at 1/16thLuma-sample precision for Luma. In addition, the Adaptive Motion Vector Resolution (AMVR) allows the Motion Vector Difference (MVD) of the CU to be coded in one of the three resolutions: Quarter-luma-sample, Integer-luma-sample, and Four-luma-sample (or 1/16 luma-sample in AFF).

Finally, the VTM5.0 inter coder introduces these new concepts: (1) the Triangular prediction (Triang) in which a CU may be further split into two triangular units, in either diagonal or inverse diagonal direction. Each of the two units is predicted using its own Uni-directional MV; (2) Combined Inter and Intra Prediction (CIIP) is proposed to improve the Intra mode in inter pictures, by combining the decided Intra mode with an extra merge indexed prediction; (3) Merge with MVD scheme (MMVD) is used for skip and merge modes with a new motion vector expression method with simplified signaling: The expression method includes starting point, motion magnitude, and motion direction; (4) Symmetric MVD (SMVD), which derives the MVD of reference list 1 from reference list 0 , based on the assumption of linear motion in bi-prediction mode.

\subsection{Quantization}

The maximum Quantization Parameter (QP) is extended from 51 to 63, and a new concept of quantization is introduced: The Dependent Quantization (DepQuant), in which the reconstruction value for a transform coefficient depends on the value of the transform coefficient that precedes it in the reconstruction order.

\subsection{Transform}

Large block-size transforms, of up to $64 \times 64$ pixels, are used. Highfrequency transform coefficients are zeroed out, so that only the lower-frequency coefficients (top-left $32 \times 32$ block) are retained. VTM5.0 uses Enhanced Multiple Transform (EMT), where two new transform matrices are added in addition to DCT-II, namely the DSTVII and the DCT-VIII. Moreover, to reduce the size of the matrices 
of transformed coefficients, a Low-Frequency Non-Separable Transform (LFNST) is applied between transform and quantization at encoder and between de-quantization and inverse transform at decoder side.

\subsection{In-loop Filtering}

Besides deblocking filter and Sample Adaptive Offset (SAO) used in HEVC, the Adaptive Loop Filter (ALF) is applied directly on the reconstructed samples of the SAO process, where one filter among 25 filters is selected for each $4 \times 4$ block, based on the direction and activity of local gradients. Nevertheless, the Luma Mapping with Chroma Scaling (LMCS) is performed before the in-loop filtering. This tool adjusts the input luma signal by redistributing it across the dynamic range using a piecewise linear mapping function and scale the chroma residuals according to the average value of the corresponding luma samples. For an inter-predicted CU, the Sub-Block Transform for inter blocks (SBT) may be used instead of EMT to code only a part of the residual block with inferred adaptive transform and the other part of the residual block is zeroed out.

\subsection{Entropy Coder}

VVC still uses the same entropy coding method used in HEVC (Context-adaptive binary arithmetic coding (CABAC)), but with some changes: The CABAC engine uses a 2 -state model with variable probability updating window sizes, instead of the pre-computed LUT of the HEVC. The transform coefficients within a Coefficient Group (CG) are coded according to pre-defined scan orders in five passes. And finally, the selected probability model and binarization models depend on the local neighborhood, where the template used to specify the local neighborhood is defined by the 5 nearby samples in the left-bottom of the current coefficient. For more information about VVC CABAC, refer to [11].

VVC is mainly targeting $4 \mathrm{~K}$ and $8 \mathrm{~K}$ video resolutions. The added coding tools compared to HEVC imply new syntax elements in the bitstream, including flags. While this is not an issue at high resolutions and high-bitrates, it may represent an unnecessary overhead at low resolutions and low-bitrates.

\section{PROPOSED METHODOLOGY}

Our aim in what follows is to identify the subset of coding tools that may be disabled in low-resolution and low-bitrate use cases, to provide a significant reduction in terms of coding complexity, while preserving compression efficiency. This can be formulated as a constrained optimization problem, which is solved using a branch-andprune approach. This technique identifies the individual tools and combination thereof that may be safely disabled, and those that have to be kept activated.

\subsection{Problem Formulation}

Consider a set of video sequences $\mathcal{V}=\left\{v_{1}, \ldots, v_{N}\right\}$. The performance of a video encoder can be measured by the rate $R$ (in Kbps) required to store the compressed videos, the resulting distortion $D$ of the decoded videos (typically measured using the weighted average $P S N R$ of the three components $Y, U$, and $V$ [3]), and the complexity $C$ of the encoding process (approximated by the run-time, measured in seconds). The values of the triple $(R, D, C)$ depend on the input video sequence $v_{n}$ and on the encoding parameter vector $\mathbf{p}=\left(p_{1}, \ldots, p_{n_{\mathrm{p}}}\right)$ of the video coder as follows

$$
(R, D, C)=f\left(v_{n}, \mathbf{p}\right),
$$

where $f$ is some (unknown) nonlinear function describing the behavior of the considered video coder. The components of $\mathbf{p}$ represent the coder input parameters, which may be adjusted to get different trade-offs between $R, D$, and $C$. The parameter vector $\mathbf{p}$ may be partitioned into subvectors. One may identify:

- $\mathbf{p}_{\mathbf{T}}$ representing binary-valued parameters indicating whether some tools are activated or remain unused;

- $\mathbf{p}_{\mathbf{C}}$ representing a finite-valued of configuration inputs for the preceding tools, e.g., the TargetBitrate and InitialQP must be specified for the Rate Control, both are integer values;

- po corresponding to other parameters which do not belong to any tool, e.g., GOP size and GOP type configurations.

To properly evaluate the performance of a coding tool, several target values of the rate $R$ have to be considered. In our work, we use the Bjontegaard Delta Rate $\left(\mathrm{BD}_{\text {rate }}\right)$ [12] to evaluate the loss of a set $\mathcal{P}_{1}=\left\{\mathbf{p}_{1}^{(1)}, \ldots, \mathbf{p}_{1}^{\left(n_{\mathrm{DR}}\right)}\right\}$ compared to another set $\mathcal{P}_{2}=$ $\left\{\mathbf{p}_{2}^{(1)}, \ldots, \mathbf{p}_{2}^{\left(n_{\mathrm{DR}}\right)}\right\}$ of values of the parameter vector. The vectors $\mathbf{p}_{j}^{(i)} \in \mathcal{P}_{j}, j=1,2$ share the same components $\mathbf{p}_{\mathrm{T}, j}, \mathbf{p}_{\mathrm{C}, j}$, and $\mathbf{p}_{\mathrm{O}, j}$, but take distinct $\mathrm{QP}$ values $\mathrm{QP}^{(i)}, i=1, \ldots, n_{\mathrm{DR}}$, with $n_{\mathrm{DR}} \geq 4$. Sets of parameter vectors $\mathcal{P}_{j}$ are called parameter configuration sets (PCS) in what follows.

Consider some reference PCS $\overline{\mathcal{P}}$, corresponding, e.g., to the best rate-distortion compromise for a set of video sequences. Our aim is to find a PCS $\mathcal{P}$ such that

$$
\begin{aligned}
& \qquad \underline{\mathcal{P}}=\arg \min _{\mathcal{P}} C(\mathcal{P}) \\
& \text { such that } \mathrm{BD}_{\text {rate }}(v, \overline{\mathcal{P}}, \mathcal{P}) \leqslant \Delta_{\text {rate }}, \\
& \quad\left(R^{(i)}, D^{(i)}, C^{(i)}\right)=f\left(v, \mathbf{p}^{(i)}\right), \mathbf{p}^{(i)} \in \mathcal{P}
\end{aligned}
$$

where $\Delta_{\text {rate }}>0$ is the largest tolerated loss in terms of $\mathrm{BD}_{\text {rate }}$ and $C(\mathcal{P})=\sum_{i=1}^{n_{\mathrm{DR}}} C^{(i)} \cdot \underline{\mathcal{P}}$ is a PCS minimizing the complexity, while keeping good compression performance compared to the optimal parameter set.

\subsection{Search for a good Parameter Configuration Set}

In what follows, we propose a method to solve the optimization problem (2) in an approximate way. Our approach concentrates on finding the subvector $\mathbf{p}_{\mathrm{T}, j}$ indicating the activated and disabled tools.

Consider $\overline{\mathbf{p}}_{\mathrm{T}}^{(i)} \in \overline{\mathcal{P}}$, the parameter vectors indicating the set of tools activated in the reference PCS. First, one builds all candidate PCS $\mathcal{P}_{1, j}=\left\{\mathbf{p}_{j}^{(1)}, \ldots, \mathbf{p}_{j}^{\left(n_{\mathrm{DR}}\right)}\right\}, j=1, \ldots, n_{1}$ with subvectors $\mathbf{p}_{\mathrm{T}, j}^{(i)}$ obtained by disabling a single tool activated in $\overline{\mathbf{p}}_{\mathrm{T}}^{(i)}$, i.e., $d_{\mathrm{H}}\left(\mathbf{p}_{\mathrm{T}, j}^{(i)}, \overline{\mathbf{p}}_{\mathrm{T}}^{(i)}\right)=1$, where $d_{\mathrm{H}}$ is the Hamming distance. Only the candidate PCS such that (3) is satisfied are further considered, the others are pruned.

Second, assuming that $n_{1}^{\prime} \leqslant n_{1}$ PCS satisfy (3), these PCS are sorted. PCS with gains in terms of $\mathrm{BD}_{\text {rate }}$ are sorted first, and then PCS with a good complexity reduction and a small $\mathrm{BD}_{\text {rate }}$ loss. Let $\mathbb{P}_{1}=\left\{\mathcal{P}_{1,1}^{\prime}, \ldots, \mathcal{P}_{1, n_{1}^{\prime}}^{\prime}\right\}$ be the ordered set of these PCS. The PCS providing a gain in terms of $\mathrm{BD}_{\text {rate }}$ (negative $\mathrm{BD}_{\text {rate }}$ ) are ordered first in $\mathbb{P}_{1}$ by decreasing $\mathrm{BD}_{\text {rate }}$ gain. Then, the PCS providing a $\mathrm{BD}_{\text {rate }}$ loss (positive $\mathrm{BD}_{\text {rate}}$ ) are ordered by decreasing value of

$$
\lambda_{j}=\frac{\left(C(\overline{\mathcal{P}})-C\left(\mathcal{P}_{1, j}\right)\right) / C(\overline{\mathcal{P}})}{\operatorname{BD}_{\text {rate }}\left(v, \overline{\mathcal{P}}, \mathcal{P}_{1, j}\right)},
$$


where the numerator of $\lambda_{j}$ is the relative complexity decrease provided by the PCS $\mathcal{P}_{1, j}$.

Third, the set $\mathbb{P}_{1}$ is split into two parts $\mathbb{P}_{2}$, containing the $n_{2} \leqslant$ $n_{1}^{\prime}$ first elements of $\mathbb{P}_{1}$ and $\mathbb{P}_{3}$ containing the remaining elements. The set $\mathbb{P}_{2}$ contains the most promising candidates PCS with a single tool disabled compared to $\overline{\mathcal{P}}$. The greedy approach presented in Algorithm 1 is then used to combine candidate PCS, i.e., disable more tools, while satisfying (3). In Algorithm 1, assuming that $\mathcal{P}_{1}=$ $\left\{\mathbf{p}_{1}^{(1)}, \ldots, \mathbf{p}_{1}^{\left(n_{\mathrm{DR}}\right)}\right\}$ and $\mathcal{P}_{2}=\left\{\mathbf{p}_{2}^{(1)}, \ldots, \mathbf{p}_{2}^{\left(n_{\mathrm{DR}}\right)}\right\}$, the notation $\mathcal{P}_{1} \wedge$ $\mathcal{P}_{2}$ corresponds to the PCS $\mathcal{P}_{3}=\mathcal{P}_{1} \wedge \mathcal{P}_{2}=\left\{\mathbf{p}_{3}^{(1)}, \ldots, \mathbf{p}_{3}^{\left(n_{\mathrm{DR}}\right)}\right\}$ such that $\mathbf{p}_{\mathrm{T}, 3}^{(i)}=\mathbf{p}_{\mathrm{T}, 1}^{(i)} \wedge \mathbf{p}_{\mathrm{T}, 2}^{(i)}$.

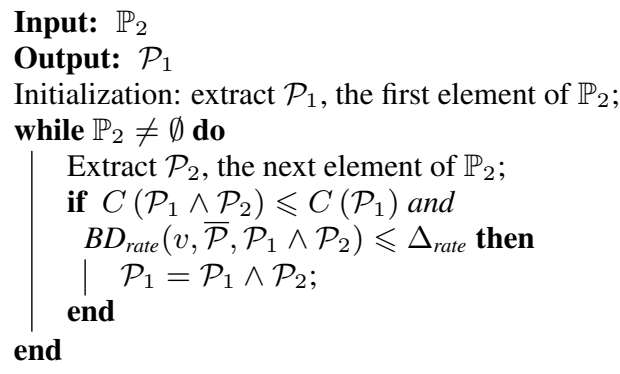

Algorithm 1: Evaluating the best PCS

Algorithm 1 progressively disable tools corresponding to the PCS in $\mathbb{P}_{2}$, starting with the most promising PCS. When disabling a tool results in a complexity reduction while satisfying (3), the PCS is updated. Tools, when disabled, do not reduce the complexity or lead to a large loss in $\mathrm{BD}_{\text {rate }}$ are kept activated.

Finally, a branch-and-prune approach presented in Algorithm 2 is considered, starting from the PCS $\mathcal{P}_{1}$ provided by Algorithm 1 to select additional tools to disable corresponding to PCS in $\mathbb{P}_{3}$. One tries first to disable a single additional tool from $\mathcal{P}_{1}$ corresponding to the various PCS in $\mathbb{P}_{3}$. All PCS $\mathcal{P} \in \mathbb{P}_{3}$ such that $\mathcal{P}_{1} \wedge \mathcal{P}$ leading to a performance decrease compared to $\mathcal{P}_{1}$ are discarded from $\mathbb{P}_{3}$. Then pairs, triples, etc. of PCS remaining in $\mathbb{P}_{3}$ are considered.

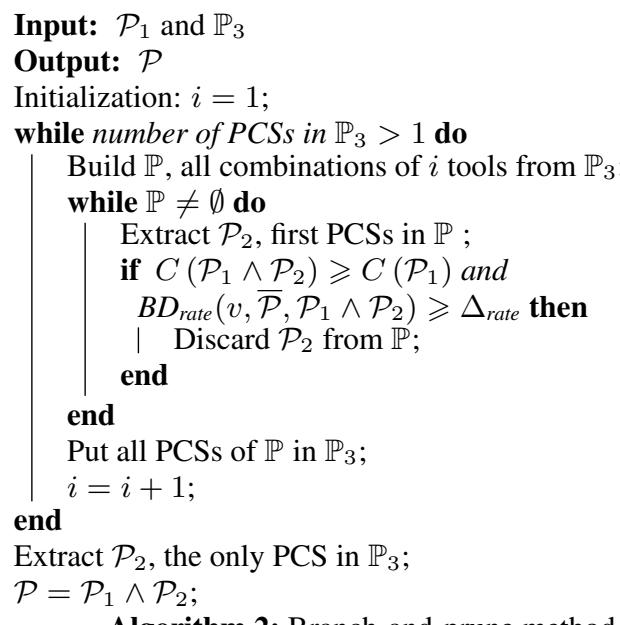

Let $\mathcal{P}_{2}$ the PCS (or combination of PCS) in $\mathbb{P}_{3}$ leading to the smallest value of $C\left(\mathcal{P}_{1} \wedge \mathcal{P}_{2}\right)$ while $\mathrm{BD}_{\text {rate }}\left(v, \overline{\mathcal{P}}, \mathcal{P}_{1} \wedge \mathcal{P}_{2}\right) \leqslant \Delta_{\text {rate }}$ Then the PCS $\mathcal{P}=\mathcal{P}_{1} \wedge \mathcal{P}_{2}$ is an approximate solution of (2).

\section{PERFORMANCE EVALUATION}

\subsection{Experimental setup}

We selected 14 JVET test sequences defined in the Common Test Conditions (CTC) [13], each one of the sequences has at most 300 images. In a first phase, 7 sequences were considered to apply our approach and identify the best PCSs. Then, in a second phase, tests are conducted on all sequences to evaluate the performance obtained with the previously identified best PCSs.

All sequences have been temporally sub-sampled at $30 \mathrm{fps}$ and spatially sub-sampled using FFmpeg [14] resulting in frames of $384 \times 216,512 \times 288$, and $640 \times 360$ pixels. A Random Access (RA) configuration is selected according to JVET CTC [13] and QP values are chosen in $\{27,32,37,42\}$. VTM5.0 is used in the experiments and run on a PC with 2 Intel Xeon CPU E5-2670 v3 24 cores @ $2.30 \mathrm{GHz}$ running under Linux. The threshold $\Delta_{\text {rate }}$ is fixed to $2 \%$ as we have noticed that this loss is subjectively unnoticeable. The value of $n_{2}$ helps to get a trade-off between complexity and accuracy in the search for $\underline{\mathcal{P}}$. Here, we take $n_{2}=n_{1}^{\prime} / 2$. The new tools of VTM5.0 we have tested in this work are listed in Table 1.

\begin{tabular}{c|c} 
DualTree & Separate Partitioning for Luma \& Chroma in I-slice \\
\hline CCLM & Chroma prediction based on linear model \\
\hline MRL & Multiple Reference Line intra prediction \\
\hline MIP & Matrix-based Intra prediction \\
\hline ISP & Intra Sub-Partitions \\
\hline CIIP & Combined Inter and Intra prediction \\
\hline SbTMVP & Sub-Pu Temporal Motion Vector Prediction \\
\hline AFF & AFFine inter motion compensation \\
\hline MMVD & Merge with MVD \\
\hline SMVD & Symmetric MVD \\
\hline Triang & inter predictions for Triangular Units \\
\hline GBI & Generalized Bi-prediction \\
\hline BDOF & Bi-Directional Optical Flow \\
\hline DMVR & Decoder Side Motion Vector Refinement \\
\hline AMVR & Adaptive MV Resolution \\
\hline EMT & Enhanced Multiple Transform \\
\hline LFNST & Low-Frequency Non-Separable Transform \\
\hline SBT & Sub-Block Transform for inter blocks \\
\hline LMCS & Luma Mapping with Chroma Scaling \\
\hline ALF & Adaptive Loop Filter
\end{tabular}

Table 1. New tools of VTM5.0 tested in this work

\subsection{Analysis}

In this section, we present experimental results in order to illustrate the proposed approach. Table 2 presents the detailed $B D_{\text {rate }}$ and complexity reduction $C$ when disabling one tool at a time for the Johnny sequence of resolutions $384 \times 216,512 \times 288$ and $640 \times 360$. These percentages are calculated relative to VTM5.0 with all tools activated (a negative $\mathrm{BD}_{\text {rate }}$ indicates a gain with respect to VTM5.0).

From Table 2, one observes that disabling tools related to interframe coding (e.g., AFF, MMVD, and Triang in resolutions $384 \times$ 216) and inloop filtering (ALF, LMCS) leads to significant gains in complexity. Other tools related to transform operations such as LFNST and EMT also lead to a significant complexity decrease. Disabling a tool can sometimes lead to an improved $\mathrm{BD}_{\text {rate }}$ when operating at low resolutions and low-bitrates, such as LMCS, SMVD 


\begin{tabular}{|c|c|c|c|c|c|c|c|c|c|c|c|}
\hline \multicolumn{4}{|c|}{ Johnny at $384 \times 216$} & \multicolumn{4}{|c|}{ Johnny at $512 \times 288$} & \multicolumn{4}{|c|}{ Johnny at $640 \times 360$} \\
\hline Disabled Tools & $B D_{\text {rate }} \%$ & $C \%$ & $\lambda$ & Disabled Tools & $B D_{\text {rate }} \%$ & $C \%$ & $\lambda$ & Disabled Tools & $B D_{\text {rate }} \%$ & $C \%$ & $\lambda$ \\
\hline LMCS & -0.42 & 10.77 & - & LMCS & -0.40 & 13.28 & - & $\mathrm{BDOF}$ & -0.29 & 13.20 & - \\
\hline SMVD & -0.01 & 8.30 & - & SMVD & 0.02 & 8.98 & 422.56 & SMVD & -0.08 & 9.67 & - \\
\hline AMVR & -0.01 & 8.46 & - & MMVD & 0.15 & 15.56 & 104.76 & LMCS & -0.07 & 9.85 & - \\
\hline GBI & 0.01 & 8.74 & 1544.07 & $\mathrm{BDOF}$ & 0.14 & 10.83 & 77.86 & MMVD & 0.10 & 15.67 & 161.51 \\
\hline CIIP & 0.03 & 7.98 & 271.58 & ISP & 0.37 & 20.55 & 55.37 & CIIP & 0.06 & 7.65 & 121.65 \\
\hline MMVD & 0.20 & 14.21 & 72.12 & CIIP & 0.14 & 7.46 & 52.16 & AMVR & 0.15 & 10.06 & 66.67 \\
\hline AFF & 0.29 & 16.59 & 57.98 & AFF & 0.58 & 19.87 & 34.48 & AFF & 0.58 & 18.72 & 32.06 \\
\hline MIP & 0.14 & 8.30 & 57.41 & AMVR & 0.26 & 8.60 & 33.43 & Triang & 0.41 & 11.39 & 28.00 \\
\hline Triang & 0.28 & 10.04 & 36.47 & Triang & 0.39 & 11.69 & 29.77 & MIP & 0.45 & 9.30 & 20.75 \\
\hline MRL & 0.20 & 7.23 & 36.01 & SBT & 0.31 & 6.72 & 21.92 & SbTMVP & 0.28 & 4.54 & 16.50 \\
\hline SbTMVP & 0.14 & 4.81 & 34.35 & MIP & 0.45 & 7.81 & 17.32 & SBT & 0.24 & 3.80 & 15.60 \\
\hline SBT & 0.07 & 1.64 & 24.45 & MRL & 0.39 & 6.38 & 16.27 & EMT & 0.42 & 6.05 & 14.49 \\
\hline EMT & 0.34 & 7.01 & 20.77 & SbTMVP & 0.32 & 5.08 & 16.04 & GBI & 0.69 & 9.71 & 14.04 \\
\hline ALF & 1.60 & 28.15 & 17.55 & GBI & 0.78 & 9.72 & 12.45 & ISP & 0.49 & 5.88 & 11.94 \\
\hline LFNST & 0.87 & 12.30 & 14.12 & LFNST & 1.07 & 12.89 & 12.03 & LFNST & 1.36 & 12.69 & 9.33 \\
\hline BDOF & 0.76 & 9.56 & 12.65 & EMT & 0.51 & 5.89 & 11.45 & ALF & 2.11 & 18.04 & 8.56 \\
\hline ISP & 0.55 & 6.35 & 11.59 & ALF & 1.98 & 20.55 & 10.38 & MRL & 0.74 & 5.81 & 7.87 \\
\hline DMVR & 0.59 & 5.30 & 8.95 & CCLM & 0.82 & 6.28 & 7.70 & CCLM & 0.88 & 5.46 & 6.18 \\
\hline CCLM & 0.79 & 5.94 & 7.50 & DMVR & 0.91 & 4.35 & 4.76 & DMVR & 0.78 & 4.48 & 5.72 \\
\hline
\end{tabular}

Table 2. $\mathrm{BD}_{\text {rate }}$ and complexity reduction $C$ in "Johnny" test sequence when disabling one tool at time

\begin{tabular}{|c|c|c|c|c|c|}
\hline Resolution & Video & $R$ Kbps & $B D_{\text {rate }} \%$ & $C \%$ & Disabled Tools \\
\hline \multirow{7}{*}{$384 \times 216$} & Johnny & $18-72$ & 1.88 & 56.06 & LMCS GBI LFNST MMVD MIP CIIP SMVD MRL SBT Triang AFF AMVR SbTMVP \\
\hline & Basketball & $48-368$ & 1.97 & 37.07 & LMCS GBI LFNST EMT MIP CIIP SMVD \\
\hline & DaylightRoad2 & $48-367$ & 2.00 & 48.91 & LMCS GBI LFNST MMVD EMT MIP CIIP MRL SBT Triang \\
\hline & BQMall & $50-331$ & 1.98 & 44.93 & LMCS GBI MMVD EMT MIP CIIP SMVD AFF CCLM \\
\hline & Drums & $89-538$ & 1.74 & 43.27 & LMCS GBI LFNST EMT MIP CIIP SMVD MRL AFF SbTMVP \\
\hline & RaceHorses & $50-403$ & 2.00 & 38.82 & LMCS GBI LFNST MMVD EMT MRL SBT \\
\hline & Kimono & $27-271$ & 1.91 & 51.21 & LMCS GBI LFNST MMVD EMT SMVD MRL AFF ISP \\
\hline Average & - & - & 1.93 & 45.75 & - \\
\hline \multirow{6}{*}{$512 \times 288$} & Johnny & $23-102$ & 1.97 & 57.01 & LMCS CIIP MMVD AFF Triang SBT ISP BIO AMVR \\
\hline & Basketball & $75-576$ & 1.81 & 35.25 & LMCS CIIP EMT LFNST SMVD MRL MIP SBT \\
\hline & BQMall & $71-477$ & 1.82 & 34.92 & LMCS CIIP EMT MMVD SMVD MRL GBI CCLM \\
\hline & Drums & $125-661$ & 2.00 & 35.25 & CIIP EMT LFNST SMVD AFF CCLM SbTMVP \\
\hline & RaceHorses & $78-763$ & 1.79 & 34.34 & LMCS CIIP EMT LFNST SMVD AFF CCLM SbTMVP \\
\hline & Kimono & $41-427$ & 1.84 & 47.78 & LMCS CIIP EMT LFNST MMVD SMVD MRL ISP AFF IMV \\
\hline Average & - & - & 1.87 & 41.85 & - \\
\hline \multirow{6}{*}{$640 \times 360$} & Johnny & $30-148$ & 1.92 & 55.24 & LMCS MIP SMVD CIIP MMVD AFF Triang AMVR BIO \\
\hline & Basketball & $99-700$ & 1.89 & 32.45 & LMCS MIP SMVD EMT LFNST SbTMVP \\
\hline & DaylightRoad2 & $100-702$ & 1.89 & 41.68 & LMCS MIP SMVD MRL GBI EMT MMVD LFNST SBT \\
\hline & BQMall & $95-639$ & 2.00 & 36.99 & LMCS MIP SMVD MRL GBI EMT MMVD CCLM \\
\hline & Drums & $164-789$ & 1.83 & 41.26 & LMCS MIP SMVD MRL GBI CIIP AFF SbTMVP \\
\hline & Kimono & $54-531$ & 1.80 & 45.77 & LMCS SMVD MRL GBI CIIP LFNST AFF Triang \\
\hline Average & - & - & 1.80 & 41.94 & - \\
\hline
\end{tabular}

Table 3. $\mathrm{BD}_{\text {rate }}$ and complexity reduction $C$ of best PCS for tested resolutions and sequences; Selected common tools are in bold

\begin{tabular}{|c|c|c|c|c|c|c|}
\hline \multirow{3}{*}{$\begin{array}{l}\text { Common Tools } \\
\text { Video }\end{array}$} & \multicolumn{2}{|c|}{$384 \times 216$} & \multirow{2}{*}{\multicolumn{2}{|c|}{$\frac{512 \times 288}{\text { LMCS CIIP MMVD SMVD EMT LFNST }}$}} & \multirow{2}{*}{\multicolumn{2}{|c|}{$\begin{array}{l}\text { 640x360 } \\
\text { LMCS MIP CIIP EMT SMVD MRL GBI MMVD }\end{array}$}} \\
\hline & LMCS GBILF & ИT MIP CIIP & & & & \\
\hline & $B D_{\text {rate }} \%$ & $C \%$ & $B D_{\text {rate }} \%$ & $C \%$ & $B D_{\text {rate }} \%$ & $C \%$ \\
\hline Johnny & 2.34 & 38.76 & 2.13 & 39.13 & 0.99 & 42.01 \\
\hline Basketball & 2.00 & 37.95 & 1.83 & 24.57 & 1.57 & 26.03 \\
\hline DaylightRoad2 & 0.68 & 38.96 & 0.94 & 35.26 & 0.98 & 35.44 \\
\hline BQMall & 1.24 & 37.81 & 1.59 & 35.73 & 1.33 & 35.31 \\
\hline Drums & 1.87 & 37.94 & 1.63 & 34.12 & 1.21 & 35.12 \\
\hline RaceHorses & 1.87 & 35.65 & 1.56 & 33.96 & 0.33 & 26.45 \\
\hline Kimono & 0.94 & 41.60 & 1.83 & 38.24 & 2.39 & 35.47 \\
\hline KristenAndSara & 1.99 & 38.62 & 1.87 & 38.93 & 0.96 & 39.32 \\
\hline CatRobot & 1.42 & 42.58 & 1.41 & 37.39 & 1.27 & 23.79 \\
\hline Tango & 1.20 & 38.55 & 1.58 & 36.06 & 0.95 & 35.18 \\
\hline ToddlerFountain & 1.20 & 38.55 & 1.58 & 36.06 & 1.43 & 39.89 \\
\hline SlideShow & 1.52 & 27.20 & 1.44 & 23.61 & 2.88 & 23.03 \\
\hline SlideEditing & 1.09 & 41.68 & 0.63 & 39.72 & 1.64 & 40.25 \\
\hline Average & 1.45 & 38.44 & 1.49 & 35.29 & 1.34 & 34.29 \\
\hline
\end{tabular}

Table 4. $\mathrm{BD}_{\text {rate }}$ and complexity reduction $C$ when disabling the common combinations of tools. Highlighted cells do not satisfy (3).

and AMVR.

Table 3 shows the $\mathrm{BD}_{\text {rate }}$ and complexity reduction $C$ for the best combination of disabled tools as obtained by the method de- scribed in Section 4.2 for seven test sequences and three resolutions. When several tools are disabled, the complexity gains accumulate in most of the cases. Nevertheless, this observation does not hold for 
$\mathrm{BD}_{\text {rate }}$, due to the complex dependency among tools and their influence on the coding efficiency. Considering Johnny at the resolution of $384 \times 216$, jointly disabling the tools: $\{$ LMCS GBI LFNST MMVD MIP CIIP SMVD MRL SBT Triang AFF AMVR SbTMVP \}, leads to a complexity reduction of $56.06 \%$, with a $\mathrm{BD}_{\text {rate }}$ loss of $1.88 \%$. For resolutions of $512 \times 288$ and $640 \times 360$, a complexity reduction of $57.01 \%$ and $55.24 \%$ is achieved with a $\mathrm{BD}_{\text {rate }}$ loss of $1.97 \%$ and $1.92 \%$ respectively. Similar results are obtained when applying the same methodology on other sequences such as, BasketballDrive, DaylightRoad2, BQMall, Drums, RaceHorses and Kimono.

These results are obtained by merely disabling tools and without algorithmic optimization. Nevertheless, the combination of tools that provide the optimal complexity reduction is different from one sequence to the other and even from one resolution to the other. The amount of complexity reduction is also varying. This is due to the spatial and temporal properties of sequences.

Yet, for each resolution, using the results of Table 3, it is possible to identify one common combination of tools satisfying constraint (3) for all sequences. Accordingly, these PCSs are: \{LMCS GBI LFNST MMVD EMT MIP CIIP $\}$ for resolution $384 \times 216,\{L M C S$ CIIP MMVD SMVD EMT LFNST $\}$ for $512 \times 288$, and $\{L M C S M I P$ CIIP EMT SMVD MRL GBI MMVD $\}$ for $640 \times 360$. Table 4 shows the $\mathrm{BD}_{\text {rate }}$ and complexity reduction $C$ for the previously identified PCSs considering the 14 test sequences. We observe that the constraint (3) is satisfied in most cases. Thus, putting these PCSs in separate profiles for each resolution will be beneficial for use cases with real-time and low-bitrate constraints. We conclude that the PCS identification approach presented in this paper provides results that are likely to be generalized to a larger set of video sequences.

\section{CONCLUSIONS}

This paper proposes an optimization of the next generation VVC targeting low-resolution video sequences encoded at low-bitrates (less than $700 \mathrm{kbps})$. The aim is to identify a set of coding tools which may be disabled while preserving coding efficiency. For this purpose, a branch-and-prune approach is proposed to determine the set of coding tools which provide the best complexity reduction, while satisfying a constraint on the $\mathrm{BD}_{\text {rate }}$ degradation.

Experimental results show that significant reduction of encoding complexity can be achieved, with negligible $\mathrm{BD}_{\text {rate }}$ loss. For instance, the joint disabling of the tools \{ LMCS MMVD AFF MIP Triang SMVD AMVR GBI CIIP MRL SbTMVP SBT LFNST $\}$ leads to a complexity reduction of $56 \%$, with a loss of $1.88 \%$ in $\mathrm{BD}_{\text {rate }}$, for Johnny at a $384 \times 216$ resolution. Moreover, a common combination of tools to disable for each resolution was determined. Our experimental results show that these common PCSs most likely cause less than $2 \% \mathrm{BD}_{\text {rate }}$ loss with good reduction in terms of encoding complexity, which is beneficial for use cases with real-time and low-bitrate constraints.

\section{REFERENCES}

[1] VNI Cisco, "Cisco visual networking index: Forecast and trends, 2017-2022," White Paper, 2018.

[2] G. Sullivan, "Deployment status of the HEVC standard," document jctvc-ab0020 of jct-vc,, JCT-VC, Torino, Italy, 2017.

[3] T. Grois D., Nguyen and Marpe D., "Performance comparison of av1, jem, vp9, and hevc encoders," in Applications of Digi- tal Image Processing XL. International Society for Optics and Photonics, 2018, vol. 10396, p. 103960L.

[4] Convenor of MPEG, "N17482: Versatile video coding project starts strongly in the joint video experts team," MPEG Press Release, 2018.

[5] X. Zhang W. Zhao L. Shen, Z. Liu and Z. Zhang, "An effective cu size decision method for hevc encoders," IEEE transactions, 2012.

[6] S. Ma L. Zhao, L. Zhang and D. Zhao, "Fast mode decision algorithm for intra prediction in hevc," in 2011 Visual Communications and Image Processing (VCIP). IEEE, 2011, pp. $1-4$.

[7] T. L. Da Silva, L. V. Agostini, and L. A. da Silva Cruz, "Fast HEVC intra prediction mode decision based on EDGE direction information," in Proc. European Signal Processing Conference, 2012.

[8] F. Sampaio, S. Bampi, M. Grellert, L. Agostini, and J. Mattos, "Motion vectors merging: low complexity prediction unit decision heuristic for the inter-prediction of HEVC encoders," in Proc. IEEE International Conference on Multimedia and Expo. IEEE, 2012, pp. 657-662.

[9] Joint Video Exploration Team, "Versatile video coding (vvc)," https://jvet.hhi.fraunhofer.de/, 2018.

[10] Convenor of JVET, "N15340: Requirements for a future video coding standard v1," in MPEG 112th, Warsaw, Poland, 2015.

[11] Y. Ye J. Chen and S. Kim, "Jvet-n1002-v1: Algorithm description for versatile video coding and test model 5 (vtm 5)," in Jvet 14th Meeting: Geneva, CH, 1927 Mar. 2019.

[12] P. Hanhart and T. Ebrahimi, "Calculation of average coding efficiency based on subjective quality scores," Journal of Visual Communication and Image Representation, vol. 25, no. 3, pp. 555-564, 2014.

[13] Teruhiko Suzuki, "Jvet-d1002 : Work plan for assessment of test material," JVET 4th Meeting, Chengdu, CN., 2016.

[14] FFmpeg Developers, "ffmpeg tool (build: ffmpeg-201807168aa6d9a-win64-static)," http://ffmpeg.org/, 2019. 